\title{
Quantum circuits for qubit fusion
}

\author{
Jonathan E. Moussa* \\ Center for Computing Research, Sandia National Laboratories, Albuquerque, New Mexico 87185, USA
}

\begin{abstract}
We consider four-dimensional qudits as qubit pairs and their qudit Pauli operators as qubit Clifford operators. This introduces a nesting, $C_{1}^{2} \subset C_{2}^{4} \subset C_{3}^{2}$, where $C_{n}^{m}$ is the $n$th level of the $m$-dimensional qudit Clifford hierarchy. If we can convert between logical qubits and qudits, then qudit Clifford operators are qubit non-Clifford operators. Conversion is achieved by qubit fusion and qudit fission using stabilizer circuits that consume a resource state. This resource is a fused qubit stabilizer state with a fault-tolerant state preparation using stabilizer circuits.
\end{abstract}

\section{INTRODUCTION}

There is increasing theoretical interest in using qudits for quantum information processing [1,2], but most experimental efforts are focused on building qubits. A natural way to relate these activities is to consider embedding qudits in qubits [3]. The simplest invertible embedding is a four-dimensional qudit in two qubits. Quantum error correction is more complicated for qudits of composite dimension, but high-distance surface codes can be constructed for qudits of any dimension [4].

This paper develops a useful physical relationship between qubits and qudits that is represented as quantum circuits. All further use of "qudit" implicitly refers to a four-dimensional qudit. We assume the availability of stabilizer operations with negligible errors on both qubits and qudits. These operations combine projective measurements in a joint Pauli basis with unitary transformations in a joint Clifford group that are able to entangle qubits with qudits. Superficially, a qudit Clifford operation can be a non-Clifford operation on two underlying qubits. To achieve actual non-Clifford operations, we need to teleport quantum states between two qubits and a qudit. We then posit the existence of a circuit element for qubit fusion,

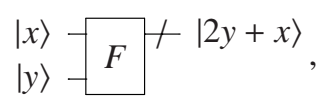

and another for the conjugate operation of qudit fission,

$$
|x\rangle+F^{\dagger}-|x \bmod 2\rangle .
$$

The relative orientation of input and output wires is used to distinguish the inequivalent qubit wires of $F$ and $F^{\dagger}$.

To implement $F$ or $F^{\dagger}$ using stabilizer operations, a qudit ancilla state must be consumed. For logical qubits and qudits encoded in different quantum codes, this state is effectively a resource for code conversion. It can be distilled and teleported to correct faulty $F$ operations analogous to gate teleportation of non-Clifford operations [5]. This combines aspects of code conversion [6] and resource state distillation [7] to implement an unconventional but universal set of quantum operations.

\footnotetext{
*godotalgorithm@gmail.com
}

We use standard quantum circuit notation [8] throughout the paper with modifications to accommodate qudits. Qudits are labeled by a slash on the left end of the wire. Pauli and Clifford gates on qudit wires denote the corresponding qudit operations. An operation controlled by a qudit in the state $|x\rangle$ is applied $x$ times. All state preparation and measurement is restricted to the qubit and qudit computational basis.

\section{STABILIZER OPERATIONS}

To enable a concise presentation of results, we assume that the reader is familiar with stabilizer operations on qubits that map between elements of the Pauli group using elements of the Clifford group [8]. The Pauli group is generated by an $i$ phase factor and an $X$ and $Z$ operator for each qubit, and the Clifford group is generated by an $\omega=\exp (i \pi / 4)$ phase factor, a controlled-NOT (CNOT) operation between qubit pairs, and a Hadamard $(H)$ and phase $(S)$ operation for each qubit.

We use the standard extension of Pauli and Clifford group structure to qudits [9] with notation similar to the qubit case. The Pauli group is still generated by a phase factor and an $X$ and $Z$ operator for each qudit, but the phase is now $\omega$ and the Pauli operator algebra on a qudit is summarized by

$$
\begin{aligned}
& Z X=i X Z, \\
& Z^{-1}=Z^{\dagger}=Z^{3}, \\
& X^{-1}=X^{\dagger}=X^{3} .
\end{aligned}
$$

Generalizations of CNOT, $H$, and $S$ operations still generate the qudit Clifford group up to a global phase [9]. They can be characterized by their action on the qudit $X$ and $Z$ operators,

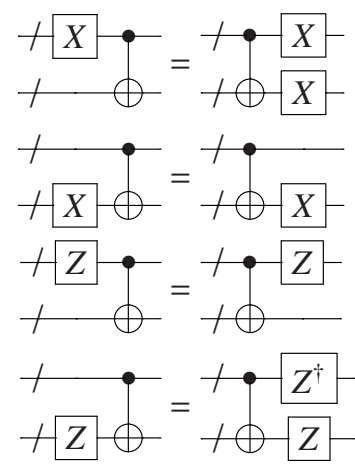




$$
\begin{aligned}
& +X-H-1 H-Z- \\
& +Z-H-=+H-X^{\dagger}- \\
& +X-S-=+S-Z-X-\times \omega \\
& +Z-S-+S-Z
\end{aligned}
$$

It was initially conjectured that other single-qudit operations would be needed to generate the qudit Clifford group [10], but CNOT, $H$, and $S$ were recently proven to be sufficient [9].

Except for the phase factor in the $S$ gate ( $\omega$ rather than $i$ ), Eq. (3) is consistent with the qubit case. $X, Z, H$, and CNOT are Hermitian and order two for qubits but are non-Hermitian and order four for qudits. This necessitates operator algebra rules that distinguish an operator from its conjugate.

We present further details of joint stabilizer operations on qubits and qudits in the next two subsections. In Sec. II A, we specify the standard representation of qudit Pauli and Clifford operations as qubit operations. In Sec. II B, we define hybrid Clifford operations between qubits and qudits.

\section{A. Standard qudit representation}

We attribute the standard representation of the qudit Pauli group [9] to the qudits generated by the $F$ gate in Eq. (1). It is typically defined by the action of Pauli and Clifford group generators on qudit computational basis states. $X$ is a "shift" operation that increments the basis state by one modulo four,

$$
|x\rangle+X-|(x+1) \bmod 4\rangle .
$$

$Z$ is a "clock" operation that shifts the phase by a power of $i$,

$$
|x\rangle+Z-i^{x}|x\rangle
$$

CNOT is a modular addition operation on the basis index,

$$
\begin{aligned}
& |x\rangle+\bullet|x\rangle \\
& |y\rangle+\mathcal{-}|(x+y) \bmod 4\rangle
\end{aligned}
$$

$H$ is a discrete Fourier transform of the quantum state,

$$
|x\rangle+H-\frac{1}{2} \sum_{y=0}^{3} i^{x y}|y\rangle
$$

$S$ is a phase shift by a power of $\omega$ with a quadratic exponent,

$$
|x\rangle+S-\omega^{x^{2}}|x\rangle \text {. }
$$

Other suggested qudit Clifford operations [10] are redundant. For example, $|x\rangle \rightarrow|3 x \bmod 4\rangle$ is the action of $H^{2}$.

We use $F$ and $F^{\dagger}$ to rewrite Eq. (4) as operations on the underlying qubits. The qudit Pauli group generators $X$ and $Z$,

$$
\begin{aligned}
& +X-=+\left.F^{\dagger}\right|^{+} \\
& +Z-=+F^{\dagger}{ }_{F}^{S},
\end{aligned}
$$

contain only qubit Clifford operations. By contrast, the qudit Clifford group generators CNOT, $H$, and $S$,

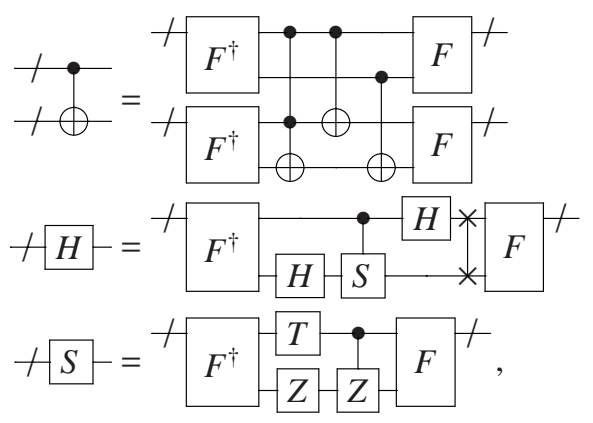

contain $T$, controlled- $S$, and Toffoli gates, which are standard qubit non-Clifford operations. If we label the $n$th level of the $m$-dimensional qudit Clifford hierarchy [5] as $C_{n}^{m}$, then it is clear from Eq. (5) that $C_{2}^{4} \subset C_{3}^{2}$. $C_{2}^{4}$ must be a strict subset of $C_{3}^{2}$ because qudit Clifford operations are not universal.

We can also use $F$ and $F^{\dagger}$ to rewrite the qubit Pauli group generators as operations on qudits. Two in particular,

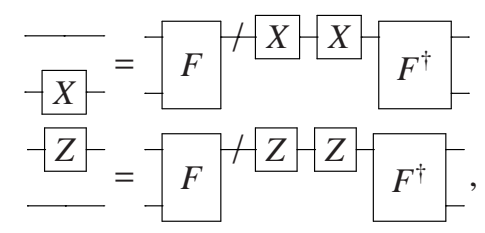

are also in the qudit Pauli group. The other two generators,

$$
\begin{aligned}
& \underline{-X}=-F, H H+X \\
& \overline{z^{2}}=-F^{/} S-S-Z^{\dagger}, F^{\dagger},
\end{aligned}
$$

are in the qudit Clifford group. We observe that $C_{1}^{2} \subset C_{2}^{4}$.

We make a final observation about fusion by decomposing the qudit Clifford operators in Eq. (7) in a qudit Pauli basis,

$$
\begin{aligned}
& X H^{2}=\frac{1}{2}\left(X+X Z^{2}+X^{\dagger}+Z^{2} X^{\dagger}\right) \\
& Z^{\dagger} S^{2}=\frac{1}{\sqrt{2}}\left(\omega^{*} Z+\omega Z^{\dagger}\right) .
\end{aligned}
$$

In the conversion between qubits and qudits, the new Pauli $Z$ operators are functions of the old Pauli $Z$ operators. This is an asymmetry in the qudit representation since the new Pauli $X$ operators are functions of both old Pauli $X$ and $Z$ operators. A complementary representation is defined in the appendix.

\section{B. Hybrid Clifford operations}

A provable construction of all Clifford operations between qudits of different dimensions is an open problem [11] that we do not attempt to solve here. We simply introduce additional generators of the Clifford group to entangle qubits and qudits that are sufficient for the purpose of this paper. We conjecture that they are sufficient to generate the entire Clifford group. 
The entangling gates that we consider are generalizations of CNOT between a qubit and qudit. They have two orientations,

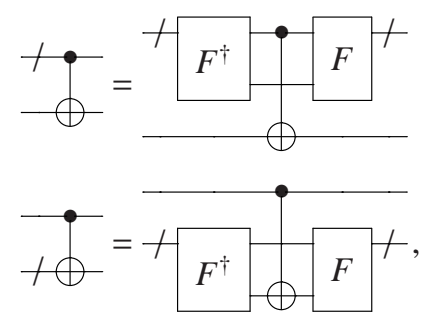

which are related by $H$ conjugation on both qubit and qudit.

For convenience, we extend the Pauli transformation rules in Eq. (3) to include the hybrid CNOT operations,

$$
\begin{aligned}
& +x=+\frac{x}{x}
\end{aligned}
$$

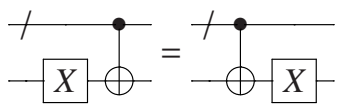

$$
\begin{aligned}
& +z \cdot \theta=+\theta \\
& +i \sigma^{-}=+\frac{1}{Z^{2}} \\
& +x=\frac{\cdot X}{10 x^{2}} \\
& \overrightarrow{+x \sigma^{-}}=\overrightarrow{+O x} \\
& +z \cdot \theta=+2 \\
& \overrightarrow{+z \sigma}=\overrightarrow{+\infty z} \text {. }
\end{aligned}
$$

By combining Eqs. (3) and (10) with standard transformation rules for qubits, we can propagate Pauli operators through any stabilizer circuit containing both qubits and qudits.

\section{CONVERSION CIRCUITS}

The qudit resource state for both fusion and fission is

$$
|F\rangle=\frac{1}{\sqrt{2}}(|0\rangle+|1\rangle),
$$

which is the fusion of a simple qubit stabilizer state

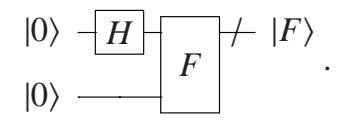

Because $|F\rangle$ is a resource state for non-Clifford operations, it should be expected that a stabilizer circuit implementation of Eq. (12) merely teleports an ancilla qudit prepared as $|F\rangle$.
It is convenient to define partial operations for fusion and fission that either prepare an input qubit in a predetermined state or measure an output qubit in a predetermined basis,

$$
\begin{aligned}
& F^{+}={ }_{|0\rangle-H Z^{-H}}{ }^{+}
\end{aligned}
$$

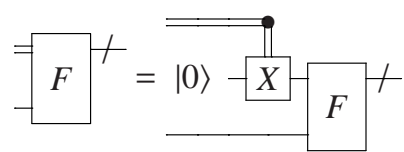

$$
\begin{aligned}
& +\sqrt[F^{\dagger}]{=}=+\sqrt{F^{\dagger}}-\sqrt{\mathrm{C}}= \\
& +F^{\dagger}=+F^{\dagger} \not \subset=
\end{aligned}
$$

A complete set of quantum states can be fused or split by these operations, but full quantum coherence is not preserved.

An advantage of the partial fusion and fission operations in Eq. (13) is that they have stabilizer circuit implementations,

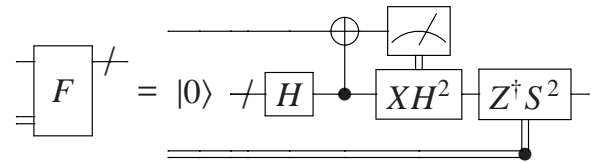

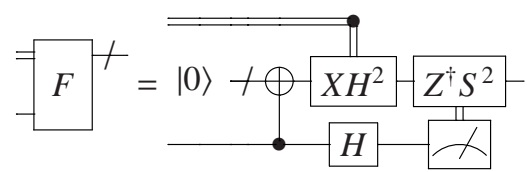

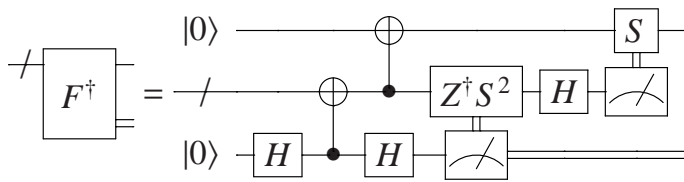

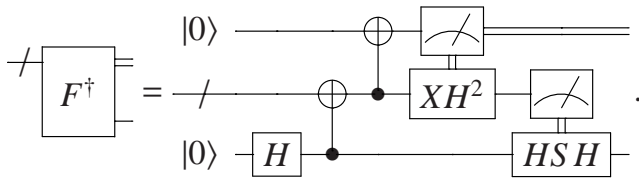

These are all standard quantum circuits for state teleportation partially rewritten using qudit Clifford operations.

With access to an ancilla qudit initialized to $|F\rangle$, stabilizer circuits for complete fusion and fission are

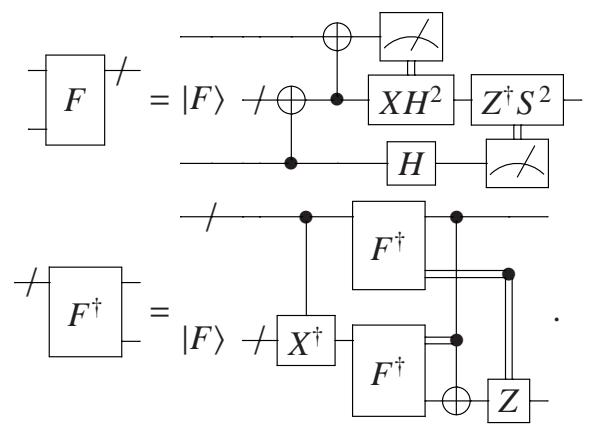

Again, these are standard circuits in nonstandard notation. 


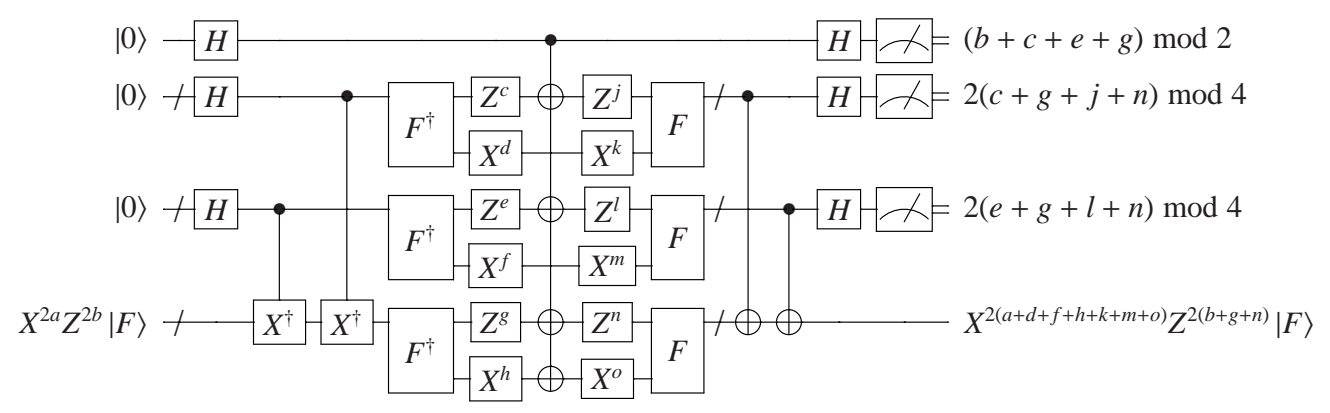

FIG. 1. $Z^{2}$ error detection circuit for twirled noisy $|F\rangle$ states with all possible error locations included.

\section{RESOURCE STATES}

Although $|F\rangle$ is an unconventional resource state, it can be used to extract the non-Clifford operations in Eq. (5) into the conventional resource states of $C_{3}^{2}$ gate teleportation [5],

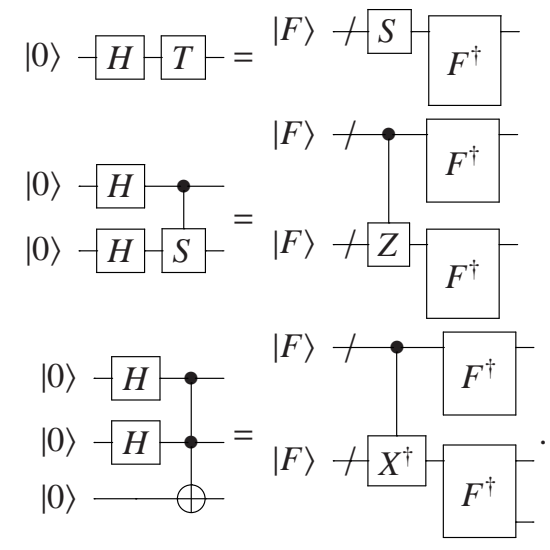

The $T$, controlled- $S$, and Toffoli gates require one, two, and three copies of $|F\rangle$ to implement respectively. Whether or not $|F\rangle$ can be prepared from finite numbers of these conventional resource states is unclear and left to future work.

Fault-tolerant state preparation of $|F\rangle$ is similar to standard magic state distillation [7]. Faulty $|F\rangle$ states are stochastically twirled using the stabilizers of $|F\rangle$ in Eq. (7),

$$
\begin{aligned}
& W_{X}(\rho)=\frac{1}{2}\left(\rho+X H^{2} \rho H^{2} X^{\dagger}\right) \\
& W_{Z}(\rho)=\frac{1}{2}\left(\rho+Z^{\dagger} S^{2} \rho S^{2} Z\right),
\end{aligned}
$$

which reduces errors to a statistical mixture of $X^{2}$ and $Z^{2}$,

$$
\begin{aligned}
\rho_{F}\left(p_{X}, p_{Z}, p_{X Z}\right)= & \left(1-p_{X}-p_{Z}-p_{X Z}\right)|F\rangle\langle F| \\
& +p_{X} X^{2}|F\rangle\left\langle F\left|X^{2}+p_{Z} Z^{2}\right| F\right\rangle\langle F| Z^{2} \\
& +p_{X Z} X^{2} Z^{2}|F\rangle\langle F| Z^{2} X^{2}
\end{aligned}
$$

These error probabilities are then reduced by applying error detection stabilizer circuits to multiple copies of $\rho_{F}$.

The detection circuit for $X^{2}$ errors is similar to Eq. (15b),

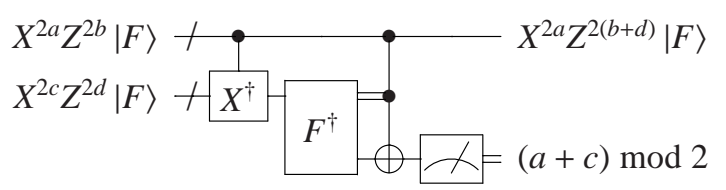

and consumes one $|F\rangle$ to detect an error in the output $|F\rangle$. On the underlying qubits, this circuit projectively measures $Z$ on the second qubit with a CNOT and measurement on the fourth qubit. It is more complicated as a qudit stabilizer circuit, with a partial fission and classically-controlled CNOT that cancel a Toffoli gate. We are unable to find a complementary circuit to detect $Z^{2}$ errors. Instead, we use a repetition code to encode a qubit $X$ measurement in Fig. 1, which consumes six $|F\rangle$.

With statistically equivalent inputs of the form in Eq. (18) and to leading order in $p_{X}, p_{Z}$, and $p_{X Z}$, Eq. (19) and Fig. 1 detect an error in $\rho_{F}$ as a nonzero measurement outcome with probability $2 p_{X}+2 p_{X Z}$ and $7 p_{Z}+7 p_{X Z}$ or otherwise output

$$
\begin{aligned}
& 2 \times \rho_{F} \rightarrow \rho_{F}\left(p_{X}^{2}+p_{X Z}^{2}, 2 p_{Z}, 2 p_{X} p_{X Z}\right) \\
& 7 \times \rho_{F} \rightarrow \rho_{F}\left(7 p_{X}, 6 p_{Z}^{3}+18 p_{Z} p_{X Z}^{2}, 6 p_{X Z}^{3}+18 p_{Z}^{2} p_{X Z}\right)
\end{aligned}
$$

respectively. For uniform error reduction, an incommensurate nesting of $X^{2}$ and $Z^{2}$ detection circuits is required. A greedy nesting that always suppresses the most probable error has a threshold of $p \approx 0.17$ for $p_{X}=p_{Z}=(1-p) p$ and $p_{X Z}=p^{2}$.

We have established that fault-tolerant $|F\rangle$ state preparation is possible with stabilizer circuits, but our construction is not efficient compared to state-of-the-art magic state distillation. Our input-output ratio for quadratic error reduction is $\approx 6.8$, which is inferior to 4 for five-dimensional qudit magic states [1] and 2 for large numbers of qubit magic states [12]. Future research should search for new $|F\rangle$ distillation protocols and a direct implementation of logical $F$ and $F^{\dagger}$ gates by converting between two qubit surface codes and a qudit surface code.

\section{CONCLUSIONS}

While qubit fusion is not an efficient method for universal quantum computation in its present form, Clifford $+F$ circuits have an advantage relative to Clifford $+T$ circuits. The cost of a quantum computation compiled into a Clifford $+T$ circuit is often measured in the number and depth of $T$ gates [13]. We can recompile Clifford $+T$ circuits into Clifford $+F$ circuits by replacing each $T$ gate with an $F$ gate using Eq. (16). We can reduce the number of $F$ gates by optimizing circuits to use two $F$ gates instead of three $T$ gates [8] for each controlled- $S$ gate and three $F$ gates instead of four $T$ gates for each Toffoli gate [14]. Thus Clifford $+F$ circuits can be more efficient than Clifford $+T$ circuits in their usage of basic resource states. 
There are two natural generalizations of qubit fusion. The first generalization is to qudit fusion, where a $p$-dimensional qudit and a $q$-dimensional qudit merge into a $p q$-dimensional qudit. Using the standard qudit Pauli and Clifford groups [9], we can attempt to generalize all the quantum circuit identities in this paper. Of particular note is the recursive construction of $H$ in Eq. (5d). $H$ is a quantum Fourier transform (QFT), and its decomposition into lower-dimensional QFTs, phase gates, and data permutations is very similar to the Cooley-Tukey fast Fourier transform algorithm [15]. The second generalization is to other pairs of Pauli and Clifford groups that operate on the same Hilbert space and have similar nesting structure. We require each Clifford group to contain both Pauli groups and have elements that are not contained within the other Clifford group. Qubit fusion results from the use of qubit and qudit Pauli groups, and magic state distillation results from the use of two qubit Pauli groups generated by $\{X, Z\}$ and $\{X S, Z\}$ [7]. Other sets of quantum operations with similar group structure might also be relevant for universal quantum computation.

Ultimately, we must judge schemes for universal quantum computation holistically. Their relative value will depend on ease of implementation on physical qubits, compatibility with quantum error correction, the threshold and code efficiency of compatible codes, an efficient method for circuit compilation, and efficient resource distillation or other implementation of a logical non-Clifford gate. These issues have been considered extensively for Clifford $+T$ circuits on qubits [16], but there are inefficiencies [17] that warrant the consideration of other schemes, such as Clifford $+F$ circuits on qubits and qudits.

\section{ACKNOWLEDGMENTS}

This work was supported by the Laboratory Directed Research and Development program at Sandia National Laboratories. Sandia National Laboratories is a multi-program laboratory managed and operated by Sandia Corporation, a wholly owned subsidiary of Lockheed Martin Corporation, for the U.S. Department of Energy's National Nuclear Security Administration under contract DE-AC04-94AL85000.

\section{Appendix: Alternate qudit representation}

It is interesting to consider other qudit representations that complement Section II A with a qubit to qudit conversion that preserves Eq. (6) and switches the role of $X$ and $Z$ in Eq. (8). We satisfy these constraints with an alternate fusion gate $G$,

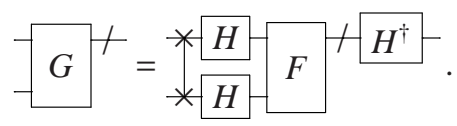

$F$ and $G$ are equivalent up to stabilizer operations. All results obtained in this paper for $F$ gates and $|F\rangle$ states generalize to this qudit representation with minor modifications of circuits. The computational basis is preserved by $F$ in Eq. (1) because it maps between $Z$ eigenstates of qubits and qudits. $G$ has the complementary effect of mapping between $X$ eigenstates.
Complementary to Eq. (5), we rewrite Eq. (4) using $G$ and $G^{\dagger}$ as a different set of operations on the underlying qubits,

$$
\begin{aligned}
& +X-=+G^{\dagger}={ }^{+}-G^{+} \\
& +Z=+G^{\dagger} \wp_{G}^{+}
\end{aligned}
$$

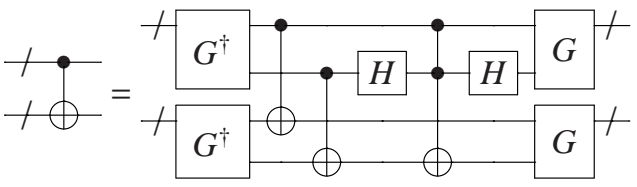

$$
\begin{aligned}
& +H-G^{\dagger}{ }_{H}^{*} S_{S} * G^{+}
\end{aligned}
$$

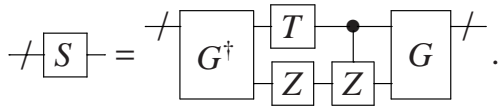

It is now $Z$ rather than $X$ that entangles the underlying qubits. The qubit operations that implement $H$ and $S$ are unchanged from Eq. (5), therefore $H$ and $S$ commute with $F G^{\dagger} . F G^{\dagger}$ is effectively a qudit non-Clifford operation. The hybrid Clifford operations of this representation are identical to Eq. (9).

By design, the mapping in Eq. (6) is unchanged for $G$,

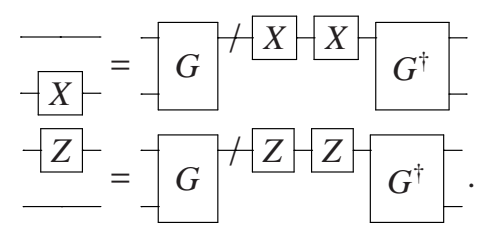

Also by design, the mapping in Eq. (7) is reversed for $G$,

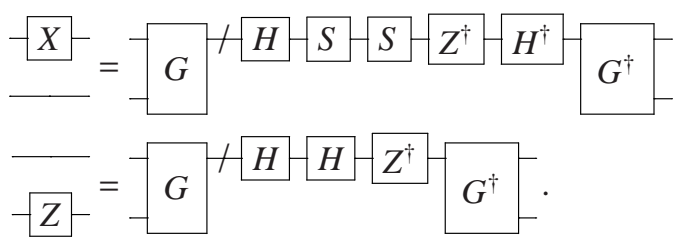

Decomposition of these operators in the qudit Pauli basis,

$$
\begin{aligned}
H^{\dagger} Z^{\dagger} S^{2} H & =\frac{1}{\sqrt{2}}\left(\omega^{*} X+\omega X^{\dagger}\right) \\
Z^{\dagger} H^{2} & =\frac{1}{2}\left(Z+X^{2} Z+Z^{\dagger}+Z^{\dagger} X^{2}\right),
\end{aligned}
$$

clearly demonstrates the complementarity with Eq. (8).

We can define $|G\rangle$ analogous to $|F\rangle$ in Eq. (12), but the two states only differ by $H$. Because of the $H$ and swAP on qubits in Eq. (A.1), the nature of the errors in a faulty $|G\rangle$ state are opposite that of $|F\rangle . Z^{2}$ errors become efficient to detect as in Eq. (19) and $X^{2}$ errors become difficult to detect as in Fig. 1. If we could switch representations and preserve the identity of errors, then $|F\rangle$ state preparation would be more efficient. This might be possible for a logical qudit encoded in a topological code, where the local identity of physical errors is decoupled from the global identity of logical errors. A logical string can be $X$-type in one spatial region and $Z$-type in another. 
[1] E. T. Campbell, H. Anwar, and D. E. Browne, Phys. Rev. X 2, 041021 (2012).

[2] F. H. E. Watson, H. Anwar, and D. E. Browne, Phys. Rev. A 92, 032309 (2015).

[3] A. Hutter, J. R. Wootton, and D. Loss, Phys. Rev. X 5, 041040 (2015).

[4] H. Bombin and M. A. Martin-Delgado, J. Math. Phys. 48, 052105 (2007).

[5] D. Gottesman and I. L. Chuang, Nature (London) 402, 390 (1999).

[6] J. T. Anderson, G. Duclos-Cianci, and D. Poulin, Phys. Rev. Lett. 113, 080501 (2014).

[7] S. Bravyi and A. Kitaev, Phys. Rev. A 71, 022316 (2005).

[8] M. A. Nielsen and I. L. Chuang, Quantum Computation and Quantum Information (Cambridge University Press, Cam- bridge, UK, 2000).

[9] J. M. Farinholt, J. Phys. A 47, 305303 (2014).

[10] D. Gottesman, Chaos Soliton Fract. 10, 1749 (1999).

[11] J. Bermejo-Vega and M. Van den Nest, Quantum Inf. Comput. 14, 181 (2014).

[12] C. Jones, Phys. Rev. A 87, 042305 (2013).

[13] M. Amy, D. R. Cheriton, D. Maslov, M. Mosca, and M. Roetteler, IEEE Trans. Comput.-Aided Des. Integr. Circuits Syst. 32, 818 (2013).

[14] C. Jones, Phys. Rev. A 87, 022328 (2013).

[15] J. W. Cooley and J. W. Tukey, Math. Comput. 19, 297 (1965).

[16] N. C. Jones, R. Van Meter, A. G. Fowler, P. L. McMahon, J. Kim, T. D. Ladd, and Y. Yamamoto, Phys. Rev. X 2, 031007 (2012).

[17] D. DiVincenzo, Phys. Rev. X 2, 030001 (2012). 